\title{
SHAPE OPTIMIZATION AND ELECTRON BUBBLES
}

\section{Pavel Grinfeld}

Department of Mathematics, Drexel University, Philadelphia, Pennsylvania, USA

\begin{abstract}
$\square \quad$ We present an analytical treatment of the shape optimization problem that arises in the study of electron bubbles. The problem is to minimize a weighted sum of a Laplace eigenvalue, volume, and surface area with respect to the shape of the domain. The analysis employs the calculus of moving surfaces and yields surprising conclusions regarding the stability of equilibrium spherical configurations. Namely, all but the lowest eigenvalue result in unstable configurations and certain combinations of parameters, near-spherical equilibrium stable configurations exist. Two-dimensional and three-dimensional problems are considered and numerical results are presented for the two-dimensional case.
\end{abstract}

Keywords Calculus of moving surfaces; Laplace eigenvalues; Shape optimization.

AMS Subject Classification 49R50.

\section{INTRODUCTION}

The problem analyzed in this paper arises in the study of electron bubbles $[1,4,12]$. It is a cleanly formulated mathematical problem that makes direct and experimentally verifiable predictions of physical phenomena [10]. The physical model, based on the principle of minimum energy, is remarkable in that it combines elements of classic physics and quantum mechanics. The resulting mathematical problem is one of shape optimization where a smooth closed surface is an independent variation. The mathematical problem can be considered in two as well as three dimensions. The two- and three-dimensional formulations are nearly identical, and the analyses parallel one another and lead to similar results. The numerical implementation is, as usual, more challenging in three dimensions.

Throughout this paper, we adhere to the indicial, rather than coordinate free, notation. This is a convenient choice because the second

Address correspondence to Pavel Grinfeld, Department of Mathematics, Drexel University, Philadelphia, PA, USA; E-mail: pg@freeboundaries.com 
variation, which is at the heart of our analysis, cannot be naturally expressed in dyadic notation.

Consider a simply connected domain $\Omega$ with a smooth boundary $S$. Suppose that the volume of this domain is $V$ and its surface area is $A$. We analyze the spectrum of the Laplace operator determined by Laplace's eigenvalue equation

$$
\nabla_{i} \nabla^{i} \psi=-\lambda \psi
$$

with zero Dirichlet boundary conditions

$$
\left.\psi\right|_{S}=0
$$

and the normalization condition

$$
\int_{\Omega} \psi^{2} d V=1 .
$$

This system specifies $\psi$ to within a sign unless $\lambda$ is a repeated eigenvaluein which case we select the corresponding $\psi$ to be real, orthogonal and each normalized in the sense of equation (1.3).

Our objective is to find local minima of the expression

$$
E=\Psi \lambda+\Sigma A+\Pi V
$$

where $\Psi$ and $\Sigma$ are positive and $\Pi$ is any real number. In physical terms, the first term is quantum effects, the second is surface tension, and the last is pressure. Unless one considers the lowest eigenvalue, one must specify how the eigenvalue is selected. When investigating possible equilibrium shapes numerically, we consider the $n$th lowest eigenvalue and follow its evolution continuously until it meets a lower eigenvalue.

The highlight of the analysis is the instability of the so-called $2 S$ state [4], which has recently been confirmed and analyzed further [13]. The $2 S$ state is the radially symmetric spherical configuration that corresponds with the second lowest such eigenvalue. It is easy to show, as we do below, that this configuration is in equilibrium, but stable only with respect to radial perturbations. It is unstable with respect to higher spherical harmonics-namely the $Y_{3 m}$. Preliminary numerical results, shown in Figure 1, indicate that there exists a near spherical equilibrium configuration that is stable. The same shape was obtained by an alternative minimization technique [13].

On a historical note, Migdal [11, 15] studied the spectrum of an electron trapped in a slightly ellipsoidal cavity and was first to analyze the perturbation of the spectrum of the Schrödinger operator induced 


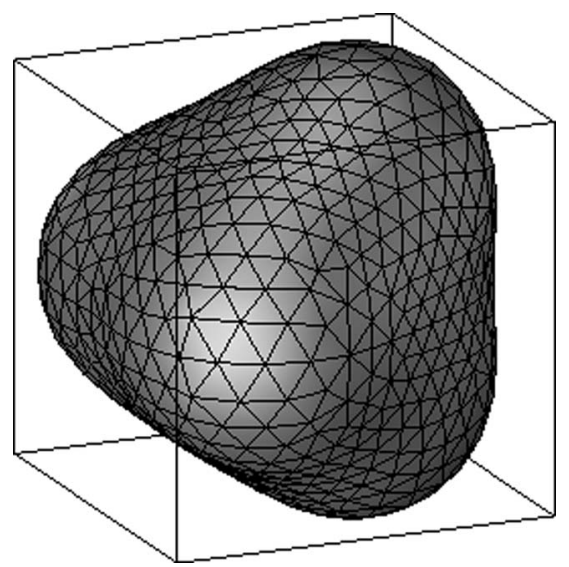

FIGURE 1 A preliminary nonspherical stable equilibrium configuration for the $2 S$ electron bubble in three dimensions.

by the deformation of the boundary. Migdal's approach is based on a change of variables that essentially converts the perturbation of the domain into a perturbation of the differential operator. Migdal's analysis can be applied to the question of stability of electron bubbles. However, our method is more general, more suitable to the analysis of the second variation, and yields a numerical recipe for discovering equilibrium stable configurations.

\section{THE CALCULUS OF MOVING SURFACES}

\subsection{The $\delta / \delta t$-Derivative}

In differential geometry on stationary manifolds, invariance is achieved by the covariant derivative $\nabla_{\alpha}$ that replaces the partial derivative $\partial / \partial \xi^{\alpha}$. On moving surfaces, the $\delta / \delta$-derivative is the key to invariance. The original prototype of this operator was proposed by Hadamard [7, 9] and subsequently significantly advanced by Thomas [16, 17], Truesdell and Toupin [18], and other authors [2].

Hadamard formulated the definition for tensor fields of order zero such as scalar fields ( $\rho$ and $C$, for example) and vector fields (position vector $\mathbf{Z}$ ). Hadamard's definition is illustrated in Figure 2. It is constructed as the limit

$$
\frac{\delta F}{\delta t}=\lim _{h \rightarrow 0} \frac{F\left(A_{t+h}\right)-F\left(A_{t}\right)}{h},
$$

where $A_{t}$ is the point at which $\delta F / \delta t$ is being evaluated, and $A_{t+h}$ is the point where the normal to $S$ at $A_{t}$ intersects $S_{t+h}$. 


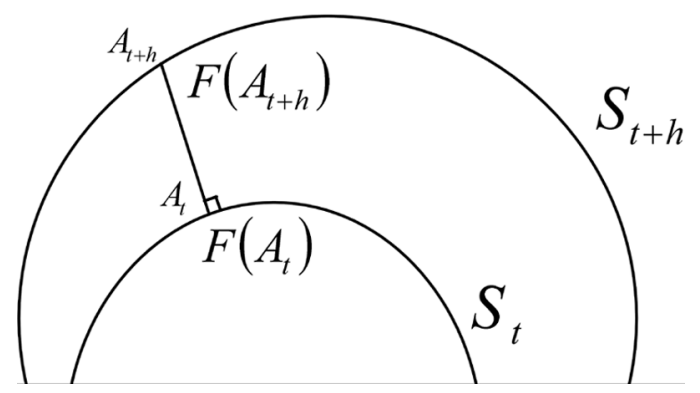

FIGURE 2 Geometric construction of the $\delta / \delta t$-derivative for $F$.

In order to express the $\delta / \delta t$-derivative analytically, suppose that surface evolution is given by the equations

$$
z^{i}=z^{i}(t, \xi)
$$

where $z^{i}$ are the coordinates in the ambient space, $\xi^{\alpha}$ are the surface coordinates, and we drop the tensor indices of function arguments. Consider the quantity $v^{i}$ defined as the partial time derivative of the parameterization scheme (2.2):

$$
v^{i}=\frac{\partial z^{i}(t, \xi)}{\partial t} .
$$

Define $v^{\alpha}$ as the projection of $v^{i}$ onto the surface:

$$
v^{\alpha}=v^{i} z_{i}^{\alpha}
$$

where $z_{i}^{\alpha}$ is the shift tensor. Then, for a scalar field $F$, the $\delta / \delta t$-derivative is defined analytically as

$$
\frac{\delta F}{\delta t}=\frac{\partial F(t, \xi)}{\partial t}-v^{\eta} \nabla_{\eta} F .
$$

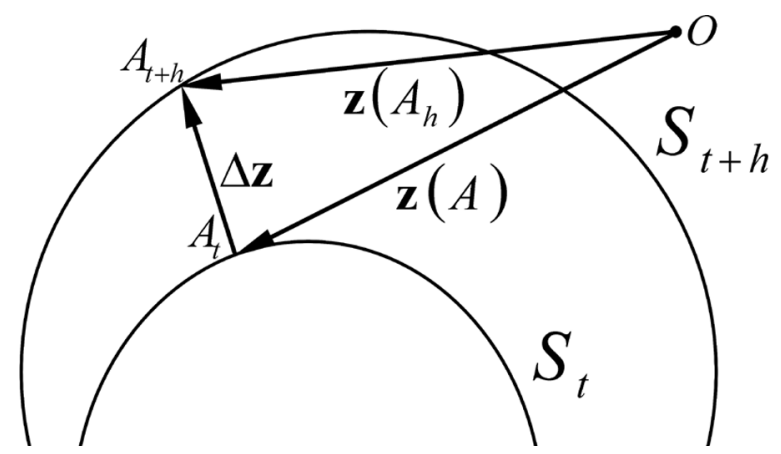

FIGURE 3 The geometric construction of $C=\lim _{h \rightarrow 0}(\mathbf{N} \cdot \Delta z) / h$. 
For a tensor $T_{j \beta}^{i \alpha}$ with a representative collection of indices, the definition, given in [2], is

$$
\frac{\delta T_{j \beta}^{i \alpha}}{\delta t}=\frac{\partial T_{j \beta}^{i \alpha}}{\partial t}-v^{\eta} \nabla_{\eta} T_{j \beta}^{i \alpha}+v^{m} \Gamma_{m k}^{i} T_{j \beta}^{k \alpha}-v^{m} \Gamma_{m j}^{k} T_{k \beta}^{i \alpha}+\nabla_{\eta} v^{\alpha} T_{j \beta}^{i \eta}-\nabla_{\beta} v^{\eta} T_{j \eta}^{i \alpha}
$$

A more detailed discussion of the $\delta / \delta t$-derivative in the context of classic tensor calculus can be found in $[2,3,6]$.

\subsection{Surface Velocity $C$}

The surface velocity $C$ is a fundamental object that captures the evolution of $S$. Introduced by Hadamard, it is defined (see Figure 3) as the normal rate of displacement in $S$ and can be expressed in terms of the $\delta / \delta t$-derivative applied to the position vector $\mathbf{Z}$ :

$$
C \mathbf{N}=\frac{\delta \mathbf{Z}}{\delta \tau}
$$

Equivalently, it can be defined in terms of the object $v^{i}$ :

$$
C=v^{i} N_{i}
$$

Interestingly, while $v^{i}$ depends on the choice of the coordinate system, its normal component $C$ is an invariant.

The quantity $C$ figures in virtually all differential relationships. If $C$ is specified at every moment of time, it completely determines the geometric evolution of the surface. In shape optimization problems, it acts as the independent variation.

\subsection{Properties of the $\delta / \delta t$-Derivative}

The following table contains the results of applying the $\delta / \delta t$-derivative to fundamental surface objects. In the following table, $B_{\beta}^{\alpha}$ is the curvature tensor, $z_{\alpha}^{i}$ is the shift tensor, $\delta_{\beta}^{\alpha}$ is the Kronecker delta, $S_{\alpha \beta}$ and $S^{\alpha \beta}$ are the covariant and the contravariant metric tensors, $N^{i}$ is the unit normal vector, and $\varepsilon_{\alpha \beta}$ and $\varepsilon^{\alpha \beta}$ are the covariant and the contravariant Levi-Civita tensors.

$$
\begin{aligned}
\frac{\delta B_{\beta}^{\alpha}}{\delta t} & =\nabla_{\beta} \nabla^{\alpha} C+C B_{\gamma}^{\alpha} B_{\beta}^{\gamma} \\
\frac{\delta z_{\alpha}^{i}}{\delta t} & =\nabla_{\alpha}\left(C N^{i}\right) \\
\frac{\delta}{\delta t} \delta_{\beta}^{\alpha} & =0
\end{aligned}
$$




$$
\begin{aligned}
\frac{\delta S_{\alpha \beta}}{\delta t} & =-2 C B_{\alpha \beta} \\
\frac{\delta S^{\alpha \beta}}{\delta t} & =2 C B^{\alpha \beta} \\
\frac{\delta N^{i}}{\delta t} & =-z_{\alpha}^{i} \nabla^{\alpha} C \\
\frac{\delta \varepsilon_{\alpha \beta}}{\delta t} & =-\varepsilon_{\alpha \beta} C B_{\gamma}^{\gamma} \\
\frac{\delta \varepsilon^{\alpha \beta}}{\delta t} & =\varepsilon^{\alpha \beta} C B_{\gamma}^{\gamma}
\end{aligned}
$$

The calculus of moving surfaces has a rule analogous to the chain rule of ordinary calculus. Namely, if $F$ is a time-dependent tensor of order zero defined in the ambient space, then the $\delta / \delta t$-derivative applied to the surface restriction of $F$ satisfies

$$
\frac{\delta F}{\delta t}=\frac{\partial F}{\partial t}+C \frac{\partial F}{\partial n} .
$$

As a consequence of this rule, we learn that the $\delta / \delta t$-derivative produces zero when applied to spatial metrics, including the metric tensor, the LeviCivita tensors, and the volume element. This rule is critical in analyzing the evolution of boundary conditions.

In problems with moving boundaries, it is often necessary to evaluate the time derivative of an integral over a deforming domain $\Omega$ or its boundary $S$. The calculus of moving surfaces provides two rules for evaluating these derivatives

$$
\begin{aligned}
\frac{d}{d t} \int_{\Omega} F d \Omega & =\int_{\Omega} \frac{\partial F}{\partial t} d \Omega+\int_{S} C F d S \\
\frac{d}{d t} \int_{S} F d S & =\int_{S} \frac{\delta F}{\delta t} d S-\int_{S} C B_{\alpha}^{\alpha} F d S .
\end{aligned}
$$

The volume rule (2.11a), in which $C$ must be taken with respect to the outward normal, is the moving surface equivalent of the fundamental theorem of calculus. The surface rule (2.11b) does not have a one-dimensional equivalent. $B_{\alpha}^{\alpha}$ is the trace of the curvature tensor $B_{\beta}^{\alpha}$. For a sphere of radius $R, B_{\alpha}^{\alpha}=-2 / R$ with respect to the outward normal. Substituting $F \equiv 1$ in equations (2.11a) and (2.11b) yields expressions for the evolution of volume $V=\int_{\Omega} d \Omega$ and surface area $A=\int_{S} d S$ :

$$
\frac{d V}{d t}=\int_{S} C d S
$$




$$
\frac{d A}{d t}=-\int_{S} C B_{\alpha}^{\alpha} d S
$$

\section{THE EQUILIBRIUM EQUATION}

Following the usual procedure in the calculus of variations, consider a family of configurations indexed by a time-like parameter $t$. All elements of the problem are functions of $t$, including the objective function $E$. The first variation of $E$ is defined as the derivative of $E$ with respect to $t$ at $t=0$

$$
\delta E=\left.\frac{d E(t)}{d t}\right|_{t=0} .
$$

Rewriting the energy $E$ as a sum of integrals

$$
E=\Psi \int_{\Omega} \nabla \psi \cdot \nabla \psi d \Omega+\Sigma \int_{S} d S+\Pi \int_{\Omega} d \Omega,
$$

we are able to apply the volume and surface identities (2.11a) and (2.11b) and obtain the following expression for the first variation of energy

$$
\delta E=-\int_{S} C\left(\Psi \nabla_{i} \psi \nabla^{i} \psi+\Sigma B_{\alpha}^{\alpha}-\Pi\right) d S .
$$

The first term in this equation is a consequence of Hadamard's formula [8] and its detailed derivation can be found in [5]. The remaining two terms are immediate consequences of identities (2.12a) and (2.12b). This expression can be used to estimate $E$ for perturbations of a configuration for which $E$ is known. For example, in [5], it was used to estimate Laplace eigenvalues of a regular $N$-sided polygon with $O\left(N^{-2}\right)$ accuracy.

Equation (3.3) yields this equation of equilibrium:

$$
\Psi \nabla_{i} \psi \nabla^{i} \psi+\Sigma B_{\alpha}^{\alpha}-\Pi=0 .
$$

The unknown quantity in this equation is the shape of the surface $S$. This equation is deeply nonlinear-a property shared by virtually all problems with unknown boundaries. No general method exists for discovering a complete set of solutions. We gain insight into this equation from two points of view. First, we consider spherical configurations that satisfy the equilibrium equation for radially symmetric eigenfunctions. Not every sphere is equilibrium-its radius must be chosen correctly. Second, 
we propose a numerical gradient descent scheme that is capable of discovering stable equilibrium configurations.

The radial stability analysis of equilibrium spherical configurations is straightforward. Stability with respect to arbitrary morphological perturbations is an entirely different story. The more general question of overall stability requires the calculation of the second variation, and it is one of the central problems in our discussion. We show that all but the lowest eigenvalue spherical configurations are unstable.

\section{RADIAL EQUILIBRIUM CONFIGURATIONS IN THREE DIMENSIONS}

\subsection{A Note on Our Variable Naming Convention}

We use the letter $\Gamma$ to denote the radius of a sphere or circle, rather than the usual choice $R$. This is because we present our analysis with electron bubbles and other physical applications in mind. We reserve the letter $R$ for the physical radius and think of $\Gamma$ as the quantity that arises when the physical problem is nondimensionalized. Likewise, we use $\gamma$, rather than $r$, for the radial coordinate.

\subsection{The Equilibrium Equation and Configuration}

Consider a sphere of radius $\Gamma$. First, note that the mean curvature is given by [14]:

$$
B_{\alpha}^{\alpha}=-\frac{2}{\Gamma} .
$$

We denote by $\psi(\gamma)$ the normalized radially symmetric eigenfunction that corresponds to $\lambda$, the $n$th lowest such eigenvalue. To make our expressions as compact as possible we omit the index $n$ but keep in mind that the entire analysis pertains to a particular choice of the eigenvalue number. The eigenfunction $\psi(\gamma)$ is given by

$$
\psi(\gamma)=\frac{\rho^{1 / 2}}{2 \Gamma} \gamma^{-\frac{1}{2}} J_{\frac{1}{2}}\left(\frac{\rho \gamma}{\Gamma}\right)
$$

where $J_{\frac{1}{2}}(x)$ is a Bessel function [19] and $\rho$ is its $n$th zero given by the simple formula

$$
\rho=n \pi
$$


The corresponding eigenvalue $\lambda$ is given by

$$
\lambda=\frac{\rho^{2}}{\Gamma^{2}}
$$

Because the eigenfunction is radially symmetric, its gradient points in the radial direction. Consequently the square of the gradient is given by

$$
\nabla^{i} \psi \nabla_{i} \psi=\psi^{\prime}(\gamma)^{2}
$$

The value of $\psi^{\prime}$ at the boundary is easily obtained from equation (4.2):

$$
\psi^{\prime}(\Gamma)=\frac{1}{2} \frac{\rho^{3 / 2}}{\Gamma^{5 / 2}} J_{-\frac{1}{2}}(\rho)=(-1)^{n} n \sqrt{\frac{\pi}{2 \Gamma^{5}}} .
$$

and consequently the equilibrium equation for a radial configuration reads

$$
\frac{n^{2} \pi \Psi}{4 \Sigma} \frac{1}{\Gamma^{5}}-\frac{1}{\Gamma}-\frac{\Pi}{2 \Sigma}=0 .
$$

This is a polynomial equation with respect to $\Gamma$ whose positive solutions correspond to equilibrium spherical configurations. Let $\Gamma_{n}$ (to distinguish it from $\Gamma$ ) be the unique solution of this equation under zero pressure, $\Pi=0$ :

$$
\Gamma_{n}=\sqrt[4]{\frac{n^{2} \pi \Psi}{4 \Sigma}}
$$

We use $\Gamma_{n}$ to introduce the dimensionless quantities $y$ and $a$

$$
y=\frac{\Gamma_{n}}{\Gamma} ; \quad a=\frac{\Pi \Gamma_{n}}{2 \Sigma}
$$

In terms of $y$ and $a$, the equilibrium equation (4.7) reads:

$$
f(y)=y^{5}-y-a=0
$$

The graph of $f(y)$ is seen in Figure 4 for several values of $a$. The minimum of $f(y)$ occurs at

$$
y_{\min }=5^{-\frac{1}{4}}
$$

and the corresponding minimum value of $f(y)$ is

$$
f\left(y_{\min }\right)=-4 \times 5^{-\frac{5}{4}}-a
$$




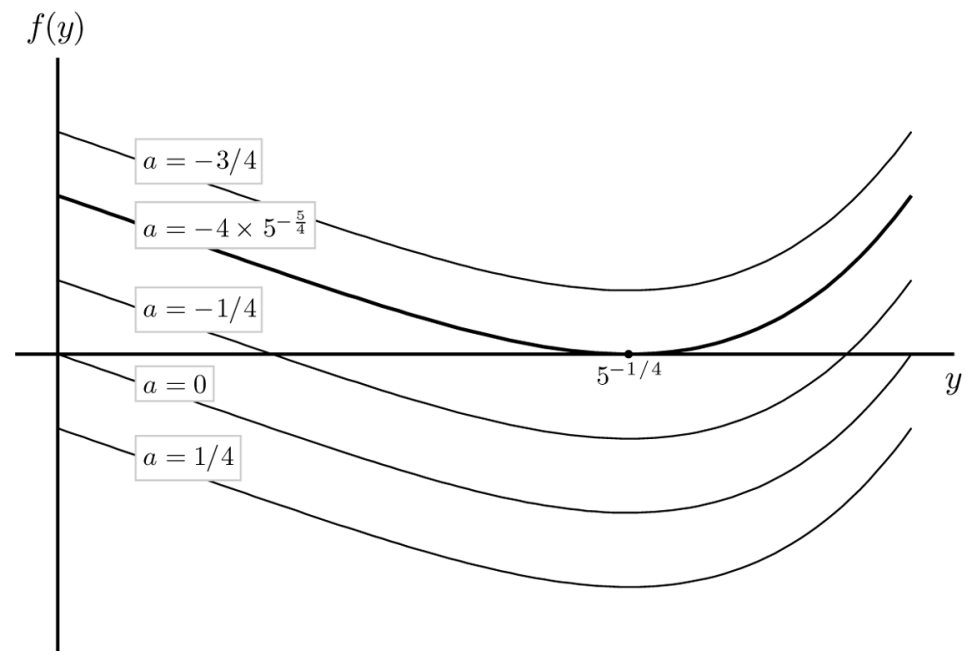

FIGURE 4 The graph of $f(y)$ defined in equation (4.10) for several values of $a$.

When $a$ is positive, there is a unique solution and, as it is greater than 1, it corresponds with an equilibrium radius below $\Gamma_{n}$. When $a$ is negative but greater than $-\frac{4}{5}\left(\frac{1}{5}\right)^{\frac{1}{4}}$, there are two solutions: one between 1 and $5^{-\frac{1}{4}}$ and the other between 0 and $5^{-\frac{1}{4}}$. Both solutions correspond to equilibrium radii greater than $\Gamma_{n}$. Finally, when $a$ is below $-4 \times 5^{-\frac{5}{4}}$, there are no solutions and therefore no equilibrium configurations.

\subsection{Radial Stability Analysis}

Note that the total energy for radial configurations is given by

$$
E(\Gamma)=\frac{n^{2} \pi^{2} \Psi}{\Gamma^{2}}+4 \pi \Gamma^{2} \Sigma+\frac{4}{3} \pi \Gamma^{3} \Pi
$$

and, in terms of the nondimensionalized parameters $a$ and $y$, we have

$$
E(y)=8 \pi \Sigma \Gamma_{n}\left(\frac{1}{2} y^{2}+\frac{1}{2} \frac{1}{y^{2}}+\frac{1}{3} \frac{a}{y^{3}}\right) .
$$

Figure 5 shows $E(\Gamma)$ as a function of $\Gamma$ for several values of $a$. The bold curve corresponds with the critical value $a=-4 \times 5^{-\frac{5}{4}}$, beyond which no equilibria exist.

Figure 5 easily reveals the conclusions regarding radial stability, and there is no need to calculate the second derivative $E^{\prime \prime}(\Gamma)$. For $a \geq 0$, there is a single stable equilibrium radius. For $-4 \times 5^{-\frac{5}{4}}<a<0$, there are two equilibrium radii-the smaller is radially stable and the larger is radially 


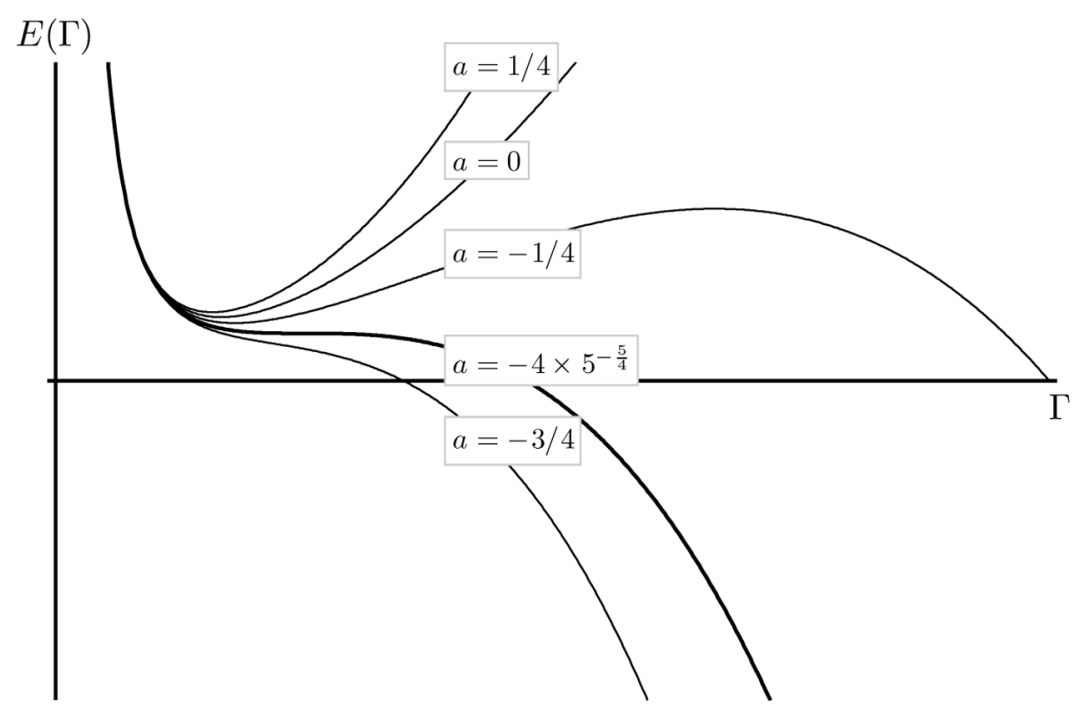

FIGURE $5 E$ as a function of radius $\Gamma$ for spherical configurations in three dimensions for several values of $a$.

unstable. For $a=-4 \times 5^{-\frac{5}{4}}$, there is a single equilibrium radius that is radially unstable. Finally, for $a<-4 \times 5^{-\frac{5}{4}}$, no equilibrium radii exist.

\section{RADIAL EQUILIBRIUM CONFIGURATIONS IN TWO DIMENSIONS}

\subsection{The Equilibrium Equation and Configuration}

Consider a circle of radius $\Gamma$. Its mean curvature is given by [14]

$$
B_{\alpha}^{\alpha}=-\frac{1}{\Gamma}
$$

Once again focusing on radially symmetric configurations, a normalized eigenfunction $\psi(\gamma)$ corresponding with the $n$th lowest radial eigenvalue is given by

$$
\psi(\gamma)=\frac{1}{\sqrt{\pi} \Gamma J_{1}(\rho)} J_{0}\left(\frac{\rho \gamma}{\Gamma}\right),
$$

where $J_{0}(x)$ and $J_{1}(x)$ are Bessel functions and $\rho$ is the $n$th root of $J_{0}(x)$. The derivative of $\psi(\gamma)$ at $\gamma=\Gamma$ is given by

$$
\psi^{\prime}(\Gamma)=-\frac{\rho}{\sqrt{\pi} \Gamma^{2}} .
$$


Consequently, the equilibrium equation (3.4) for a circle of radius $\Gamma$ is

$$
\frac{\rho^{2} \Psi}{\pi \Sigma} \frac{1}{\Gamma^{4}}-\frac{1}{\Gamma}-\frac{\Pi}{\Sigma}=0
$$

Following the three-dimensional case, define $\Gamma_{n}$ as the equilibrium radius at $\Pi=0$ :

$$
\Gamma_{n}=\sqrt[3]{\frac{\rho^{2} \Psi}{\pi \Sigma}} .
$$

The dimensionless parameters $y$ and $a$ are defined analogously to equation (4.9):

$$
y=\frac{\Gamma_{n}}{\Gamma} ; \quad a=\frac{\Pi \Gamma_{n}}{\Sigma} .
$$

The dimensionless equilibrium equation is

$$
f(y)=y^{4}-y-a=0 .
$$

The minimum of $f(y)$ occurs at

$$
y_{\min }=4^{-\frac{1}{3}}
$$

where $f(y)$ attains the value

$$
f\left(y_{\min }\right)=-3 \times 4^{-\frac{4}{3}}-a .
$$

The plot of $f(y)$ for several values of $a$ can be seen in Figure 6 .

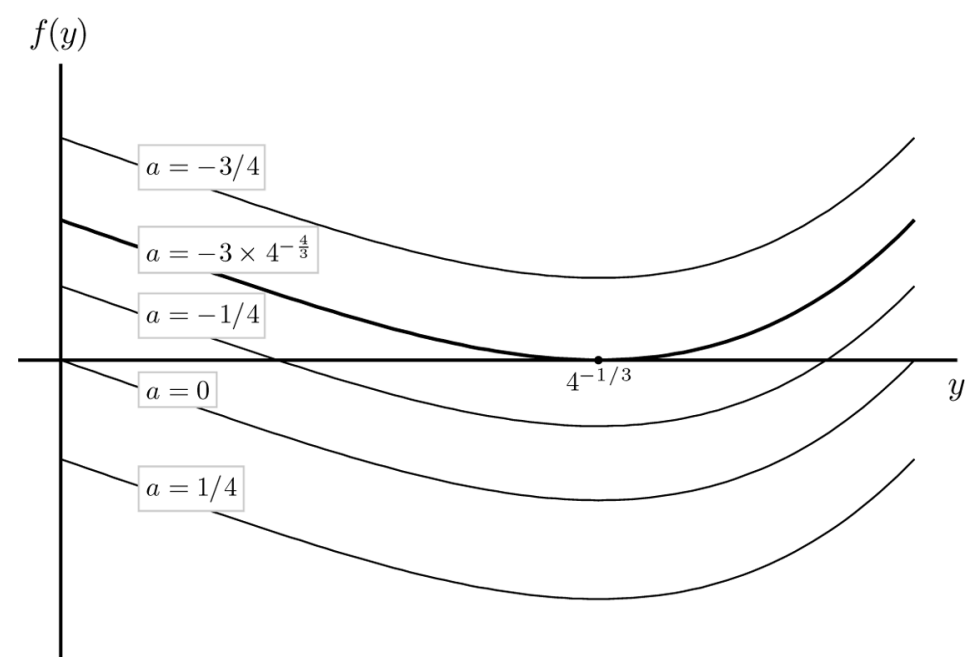

FIGURE 6 The graph of $f(y)$ defined in equation (5.7) for several values of $a$. 


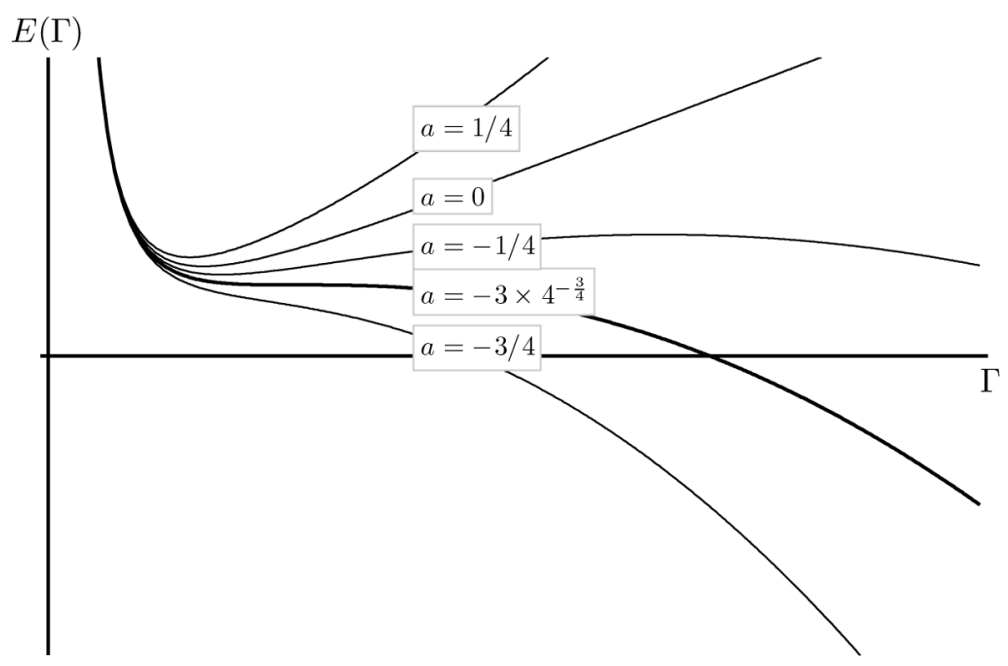

FIGURE $7 \quad E$ as a function of radius $\Gamma$ for spherical configurations in two dimensions for several values of $a$.

The total energy $E$ as a function of $\Gamma$ is given by

$$
E(\Gamma)=\frac{\rho^{2} \Psi}{\Gamma^{2}}+2 \pi \Gamma \Sigma+\pi \Gamma^{2} \Pi
$$

or, in dimensionless terms,

$$
E(y)=2 \pi \Sigma \Gamma_{n}\left(\frac{1}{2} y^{2}+\frac{1}{y}+\frac{1}{2} \frac{a}{y^{2}}\right) .
$$

The plot of $E(\Gamma)$ is seen in Figure 7 . We reach conclusions analogous to those in three dimensions: For $a \geq 0$, there is a single stable equilibrium radius. For $-3 \times 4^{-\frac{4}{3}}<a<0$, there are two equilibrium radii-the smaller is radially stable and the larger is radially unstable. For $a=-3 \times 4^{-\frac{4}{3}}$, there is a single equilibrium radius and it is radially unstable. Finally, for $a<-3 \times 4^{-\frac{4}{3}}$, no equilibrium radii exist.

Figure 8 contains a gallery of equilibrium radially stable configurations for $n=1,2,3$, and $\Pi=-0.1,0$, and 0.1 .

\section{THE SECOND VARIATION CALCULATION}

\subsection{Common Part of the Analysis}

An equilibrium configuration is unstable if there exists a morphological perturbation $C$ for which the second variation of $E$ is negative. This section is devoted to computing the second variation. 

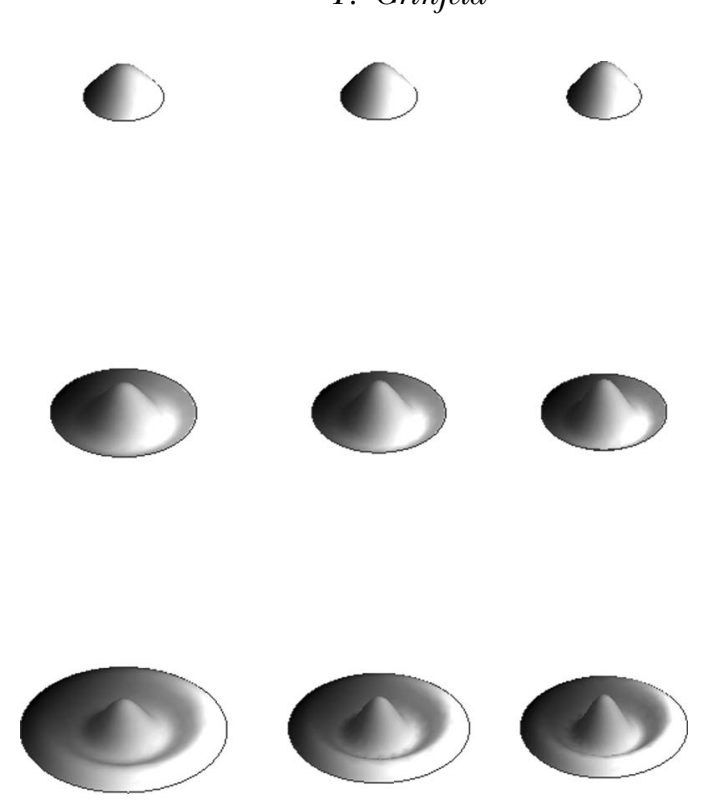

FIGURE 8 Equilibrium $1 S, 2 S$, and $3 S$ configurations in two dimensions for $\Pi=-0.1,0.0$, and 0.1 , drawn to scale.

Apply the rule for differentiating surface integrals (2.11b) to the first variation of energy in equation (3.3). The result is

$$
\delta^{2} E=-\int_{S} C\left(2 \Psi \nabla^{i}\left(\delta \psi+C N^{j} \nabla_{j} \psi\right) \nabla_{i} \psi+\Sigma\left(\nabla^{\alpha} \nabla_{\alpha} C+C B_{\beta}^{\alpha} B_{\alpha}^{\beta}\right)\right) d S,
$$

where $\nabla_{\alpha}$ is the surface gradient, and the variation of the eigenfunction $\delta \psi$ is defined as $\delta \psi=\partial \psi /\left.\partial t\right|_{t=0}$. This expression applies to arbitrary equilibrium configurations and arbitrary perturbations $C$ of the interface, as long as both are sufficiently smooth.

When the equilibrium configuration is a sphere or a circle and $\psi$ is radially symmetric, its gradient points in the radial direction. Although its perturbation $\delta \psi$ is not radially symmetric, the nonradial components of $\nabla^{i} \delta \psi$ are irrelevant as $\nabla^{i} \delta \psi$ is contracted with $\nabla_{i} \psi$. Therefore, $\delta^{2} E$ can be rewritten as

$$
\delta^{2} E=-\int_{S} C\left(2 \Psi\left(\frac{\partial}{\partial \gamma} \delta \psi+\psi^{\prime \prime}(\Gamma)\right) \psi^{\prime}(\Gamma)+\Sigma\left(\nabla^{\alpha} \nabla_{\alpha} C+C B_{\beta}^{\alpha} B_{\alpha}^{\beta}\right)\right) d S .
$$

Note that the third ground form $B_{\beta}^{\alpha} B_{\alpha}^{\beta}$ has different values in two and three dimensions

$$
B_{\beta}^{\alpha} B_{\alpha}^{\beta}= \begin{cases}2 \Gamma^{-2}, & \text { in } 3 \mathrm{D} \\ \Gamma^{-2}, & \text { in } 2 \mathrm{D}\end{cases}
$$


The only truly challenging term in (6.2) is the one containing the eigenfunction perturbation $\delta \psi$. The eigenfunction perturbation $\delta \psi$ is calculated by solving the perturbed boundary value problem (1.1)(1.3). The perturbation of the eigenvalue problem (1.1)-(1.3) is obtained by applying the partial time derivative to the bulk equation (1.1), the $\delta / \delta t$-derivative to the boundary condition (1.2), and the ordinary time derivative to the normalization condition (1.3). The result is the following boundary value problem

$$
\begin{aligned}
\nabla_{i} \nabla^{i} \delta \psi & =-\delta \lambda \psi-\lambda \delta \psi \\
\left.\delta \psi\right|_{\partial \Omega} & =-C N^{i} \nabla_{i} \psi \\
2 \int_{\Omega} \psi \delta \psi d \Omega+\int_{S} C \psi^{2} d \Omega & =0 .
\end{aligned}
$$

This system can be simplified in two aspects. First, for radially symmetric configurations, the normal derivative coincides with $d / d \gamma$ :

$$
N^{i} \nabla_{i} \psi=\psi^{\prime}(\Gamma)
$$

Second, due to zero Dirichlet boundary conditions (1.2), the second integral in the normalization equation vanishes. Therefore, the resulting boundary value problem for $\delta \psi$, reads

$$
\begin{aligned}
\nabla_{i} \nabla^{i} \delta \psi+\lambda \delta \psi & =-\delta \lambda \psi \\
\left.\delta \psi\right|_{S} & =-C \psi^{\prime}(\Gamma) \\
\int_{\Omega} \psi \delta \psi d \Omega & =0 .
\end{aligned}
$$

In the following sections, we employ Fourier methods to solve this system in two and three dimensions and use those solutions to calculate the second variation of $E$.

\subsection{The Second Variation in Three-Dimensions}

Decompose $C$ with respect to spherical harmonics $Y_{l m}(\theta, \phi)$ :

$$
C=\Gamma \sum_{l, m} C_{l m} Y_{l m}(\theta, \alpha) .
$$

We solve system $(6.6 \mathrm{a})-(6.6 \mathrm{c})$ by decomposing $\delta \psi$ in spherical harmonics

$$
\delta \psi(\gamma, \theta, \phi)=\sum_{l, m} s_{l m}(\gamma) Y_{l m}(\theta, \phi)
$$


The ordinary differential equation that results for $s_{l m}(\gamma)$ is easily solved:

$$
\begin{aligned}
& s_{00}(\gamma)=-\frac{C_{00}}{4 \sqrt{4 \pi}} \rho^{1 / 2} \Gamma^{-1} \gamma^{-1 / 2}\left(J_{\frac{1}{2}}\left(\frac{\rho \gamma}{\Gamma}\right)+2 \rho \frac{\gamma}{\Gamma} J_{-\frac{1}{2}}\left(\frac{\rho \gamma}{\Gamma}\right)\right) \\
& s_{l m}(\gamma)=-\frac{C_{l m}}{2} \rho^{3 / 2} \Gamma^{-1} \frac{J_{-\frac{1}{2}}(\rho)}{J_{l+\frac{1}{2}}(\rho)} \gamma^{-1 / 2} J_{l+\frac{1}{2}}\left(\frac{\rho \gamma}{\Gamma}\right) .
\end{aligned}
$$

We now turn to the remaining terms in equation (6.2). Because the spherical harmonics $Y_{l m}(\theta, \alpha)$ are eigenfunctions of the surface Laplacian on the unit sphere with eigenvalues $-l(l+1)$, we have

$$
\nabla^{\alpha} \nabla_{\alpha} C=-\frac{1}{\Gamma} \sum_{l, m} l(l+1) C_{l m} Y_{l m}(\theta, \alpha) .
$$

Finally, the ordinary derivatives of the unperturbed eigenfunction $\psi$ are easily obtained from equation (4.2):

$$
\begin{aligned}
& \psi^{\prime}(\Gamma)=\frac{1}{2} \rho^{3 / 2} \Gamma^{-5 / 2} J_{-\frac{1}{2}}(\rho) \\
& \psi^{\prime \prime}(\Gamma)=-\rho^{3 / 2} \Gamma^{-7 / 2} J_{-\frac{1}{2}}(\rho) .
\end{aligned}
$$

Putting all of the ingredients together, we obtain the following expression for the second variation:

$$
\begin{gathered}
\delta^{2} E=\Sigma \Gamma^{2}\left(2 C_{00}^{2}\left(5 y^{4}-1\right)+\sum_{l \neq 0, m}\left(4 y^{4}\left(\frac{\rho_{n} J_{l-\frac{1}{2}}(\rho)}{J_{l+\frac{1}{2}}(\rho)}-(l-1)\right)\right.\right. \\
\left.+(l+2)(l-1))\left|C_{l m}\right|^{2}\right) .
\end{gathered}
$$

\subsection{The Second Variation in Two-Dimensions}

Decompose $C$ as a Fourier series

$$
C=\Gamma \sum_{l} C_{l} e^{i l \theta} .
$$

Analogously to the three-dimensional case, we solve the perturbed system (6.6a)-(6.6c) by decomposing $\delta \psi$ as a Fourier series

$$
\delta \psi(\gamma, \theta)=\sum_{l=-\infty}^{\infty} s_{l}(\gamma) e^{i l \theta}
$$


and find that

$$
\begin{aligned}
& s_{0}(\gamma)=\frac{C_{0}}{\sqrt{\pi \Gamma^{2}} J_{1}(\rho)}\left(J_{1}\left(\frac{\rho \gamma}{\Gamma}\right) \frac{\rho \gamma}{\Gamma}-J_{0}\left(\frac{\rho \gamma}{\Gamma}\right)\right), \\
& s_{l}(\gamma)=\frac{C_{l} \rho}{\sqrt{\pi \Gamma^{2}} J_{l}(\rho)} J_{l}\left(\frac{\rho \gamma}{\Gamma}\right) .
\end{aligned}
$$

Combining all the terms, we arrive at the following expression for the second variation

$$
\delta^{2} E=\Sigma \Gamma\left(\left(4 y^{3}-1\right) C_{0}^{2}+\cdots \sum_{l \neq 0}\left(2 y^{3}\left(\frac{\rho J_{l}^{\prime}(\rho)}{J_{l}(\rho)}+1\right)+(l+1)(l-1)\right)\left|C_{l}\right|^{2}\right) .
$$

The problem of eigenvalue perturbation comes up in other contexts [13]. We would therefore like to include a general formula for the second eigenvalue variation $\delta^{2} \lambda$ induced by evolution of the interface $C$ on the unit circle:

$$
\delta^{2} \lambda \frac{\lambda}{\pi}\left(-\int_{S} \frac{\delta C}{\delta \tau} d S+2 \int_{S} C\left(C_{0}+\sum_{m \neq 0} C_{m} \frac{\rho J_{m}^{\prime}(\rho)}{J_{m}(\rho)} e^{i m \alpha}\right) d S+\int_{S} C^{2} d S\right)
$$

This expression can be partially rewritten as an infinite series

$$
\delta^{2} \lambda=-\frac{\lambda}{\pi} \int_{S} \frac{\delta C}{\delta \tau} d S+4 \lambda\left(C_{0}^{2}+\sum_{m \neq 0}\left|C_{m}\right|^{2} \frac{\rho J_{m}^{\prime}(\rho)}{J_{m}(\rho)}\right)+2 \lambda \sum_{m=-\infty}^{\infty}\left|C_{m}\right|^{2} .
$$

\section{STABILITY ANALYSIS}

\subsection{Stability in Three-Dimensions}

The expression (6.12) holds the key to the stability criteria for the equilibrium spherical configurations. The term that contains $C_{00}$ is responsible for radial variations and yields the following stability criterion

$$
5 y^{4}-1>0
$$

which, as expected, is completely equivalent to the conclusions obtained in the section of radial stability. The criteria for stability against higher harmonics reads (recall that $\rho=n \pi$ ):

$$
P_{n}^{l}=4 y^{4}\left(\frac{n \pi J_{l-\frac{1}{2}}(n \pi)}{J_{l+\frac{1}{2}}(n \pi)}-(l-1)\right)+(l+2)(l-1)>0,
$$


TABLE $1 P_{n}^{l}$ for the 6 lowest eigenvalues and 10 lowest spherical harmonics in three dimensions*

\begin{tabular}{rcrrrrr}
\hline$l$ & $\lambda_{1}$ & $\lambda_{2}$ & \multicolumn{1}{c}{$\lambda_{3}$} & $\lambda_{4}$ & \multicolumn{1}{c}{$\lambda_{5}$} & \multicolumn{1}{c}{$\lambda_{6}$} \\
\hline 1 & 0 & 0 & 0 & 0 & 0 & 0 \\
2 & 13.16 & 52.64 & 118.44 & 210.55 & 328.99 & 473.74 \\
3 & 25.08 & $-\mathbf{1 7 . 3 5}$ & $-\mathbf{1 2 . 4 4}$ & $-\mathbf{1 1 . 2 6}$ & $-\mathbf{1 0 . 7 8}$ & $\mathbf{- 1 0 . 5 3}$ \\
4 & 38.13 & 19.34 & 39.49 & 67.24 & 102.82 & 146.27 \\
5 & 52.79 & 39.87 & 578.06 & $-\mathbf{8 8 . 1 1}$ & $-\mathbf{5 2 . 9 1}$ & $\mathbf{- 4 2 . 5 2}$ \\
6 & 69.26 & 59.17 & 17.28 & 37.53 & 56.25 & $\mathbf{7 7 . 7 0}$ \\
7 & 87.60 & 79.24 & 55.97 & 103.30 & 280.61 & $\mathbf{- 9 6 7 . 0 6}$ \\
8 & 107.87 & 100.68 & 83.77 & $-\mathbf{1 4 7 . 9 2}$ & 21.29 & 47.38 \\
9 & 130.08 & 123.75 & 110.17 & 65.80 & 100.50 & 146.78 \\
10 & 154.25 & 148.59 & 137.12 & 110.16 & 197.34 & $\mathbf{- 3 1 2 . 6 1}$ \\
\hline
\end{tabular}

*The negative values, indicating instability against a particular harmonic, are emphasized in bold.

where $y$ is determined by equation (4.10) and only the larger root, being radially stable, is of interest.

First note that as $J_{\frac{1}{2}}(n \pi)=0, P_{n}^{1}$ vanishes indicating neutral stability with respect to rigid translations. This is to be expected as the total energy of the physical system depends only on the shape and is therefore translationally invariant.

The numerical values for $P_{n}^{l}$ are given in Table 1 for $\Pi=0$.

This table leads to the conclusion that only the "ground state" $n=1$ is stable and that the rest are unstable against any of the third harmonics $Y_{3 n}(\theta, \phi)$.

\subsection{Stability in Two-Dimensions} reads

We perform a similar analysis on expression (6.16). Radial stability

$$
4 y^{3}-1>0
$$

which is entirely consistent with the conclusions obtained in the section on radial stability. The criteria for stability against higher harmonics reads:

$$
P_{n}^{l}=2 y^{3}\left(\frac{\rho J_{l}^{\prime}(\rho)}{J_{l}(\rho)}+1\right)+(l+1)(l-1)
$$

where $y$ is determined by equation (5.7) and only the larger root, being radically stable, is of interest. Translational stability of neutral and for higher harmonics, the value of $P_{n}^{l}$ is contained in Table 2 .

This table leads to the same conclusions as in the three-dimensional case: the ground state is stable while the rest are unstable against the third harmonic. 
TABLE $2 P_{n}^{l}$ for the 6 lowest eigenvalues and 10 lowest spherical harmonics in two dimensions*

\begin{tabular}{rrrrrrr}
\hline$l$ & $\lambda_{1}$ & $\lambda_{2}$ & \multicolumn{1}{c}{$\lambda_{3}$} & $\lambda_{4}$ & \multicolumn{1}{c}{$\lambda_{5}$} & \multicolumn{1}{c}{$\lambda_{6}$} \\
\hline 1 & 0 & 0 & 0 & 0 & 0 & 0 \\
2 & 6.78 & 31.47 & 75.89 & 140.04 & 223.93 & 327.56 \\
3 & 14.44 & $-\mathbf{1 . 4 2}$ & $-\mathbf{0 . 4 8}$ & $-\mathbf{0 . 2 4}$ & $-\mathbf{0 . 1 5}$ & $\mathbf{- 0 . 1 0}$ \\
4 & 23.78 & 16.00 & 27.18 & 43.24 & 64.22 & 90.13 \\
5 & 35.00 & 29.54 & $-\mathbf{1 2 1 . 5 2}$ & $-\mathbf{1 4 . 5 0}$ & $-\mathbf{6 . 7 4}$ & $\mathbf{- 4 . 0 6}$ \\
6 & 48.15 & 43.85 & 26.90 & 36.01 & 45.87 & 57.61 \\
7 & 63.26 & 59.69 & 49.56 & 78.83 & 320.49 & $\mathbf{- 1 1 5 . 7 0}$ \\
8 & 80.35 & 77.27 & 69.74 & 11.49 & 45.52 & 56.27 \\
9 & 99.41 & 96.71 & 90.60 & 72.00 & 89.14 & 116.82 \\
10 & 120.47 & 118.05 & 112.86 & 100.86 & 163.08 & 3.33 \\
\hline
\end{tabular}

*The negative values, indicating instability against a particular harmonic, are emphasized in bold.

\section{NUMERICAL INVESTIGATION OF EQUILIBRIUM STABLE SHAPES}

The stable equilibrium shapes can be discovered numerically by implementing the gradient descent scheme

$$
C=\Psi \nabla^{i} \psi \nabla_{i} \psi+\Sigma B_{\alpha}^{\alpha}-\Pi
$$

Alternatively, one could use a black box optimizer, which can be applied directly to the objective function (1.4) thereby bypassing the entire analysis pertaining to moving surfaces. This approach may be advisable in three dimensions where computation of the mean curvature $B_{\alpha}^{\alpha}$ for a triangulated surface is not straightforward.

Note that, as a direct consequence of the Dirichlet boundary condition (1.2), the gradient $\nabla^{i} \psi$ points strictly in the normal direction. Therefore, $\nabla^{i} \psi \nabla_{i} \psi=(\partial \psi / \partial n)^{2}$. The normal derivatives can be determined in the finite element method by using Lagrange multipliers to enforce Dirichlet boundary conditions.

Preliminary results for the three-dimensional case were described in $[4,13]$ and the research on this topic continues today. We therefore concentrate on the two-dimensional case.

\subsection{Equilibrium Stable Shapes in Two-Dimensions}

We begin with the radially symmetric $S$ states, as these are the primary object of this paper. Figure 9 shows equilibrium stable configurations that result for the first three radially symmetric eigenvalues. While in the first two simulations we took $\Pi=0$, in the third one we used $\Pi=-0.1$ in order to the prevent the eigenvalue from crossing its next-lowest neighbor in the course of the evolution. 

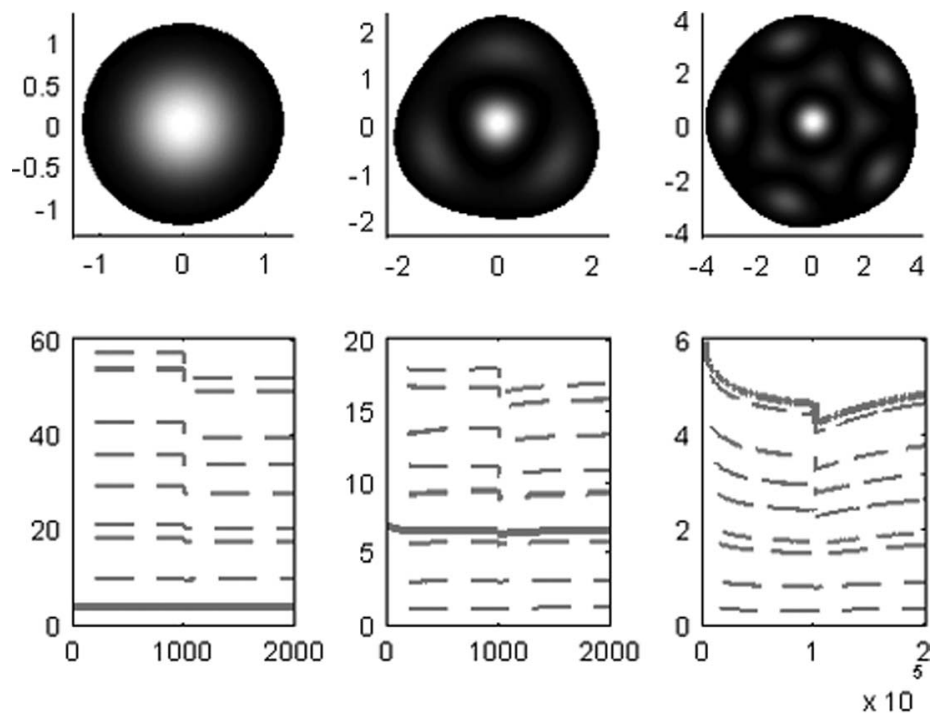

FIGURE 9 Equilbrium spherical configurations for the three lowest spherically symmetric eigenvalues. The bottom charts show the evolution of the eigenvalues against the number of iterations. In each of the cases, the mesh was refined once halfway through the simulation.

The plots below the final configurations indicate the evolution of the eigenvalues as a function of number of iteration taken. The objective eigenvalue is shown in bold. In each of the three cases, the mesh was refined halfway through the iteration. The refinement of the mesh is manifested by all eigenvalues dropping as a finer mesh is able to deliver a lower minimum to the Rayleigh quotient. After the refinement, the eigenvalues increase. That is because the perimeter, having doubled its degrees of freedom, is now able to shrink further and the reduction in the perimeter turns out to be a stronger effect than the increase in the eigenvalue.

The eventual location and orientation of the equilibrium shape are due to the specifics of the mesh that essentially lifts the symmetry of the original configuration. The initial mesh contains 144 nodes and 254 triangles while the refined mesh contains 541 points and 1016 triangles. The Laplace eigenvalue problem is solved by the FEM method with linear elements. The evolution in time is carried out by a forward Euler scheme. The normal at each node is computed as a weighted average of normals to the adjacent sides with weights inversely proportional to the side length. As previously mentioned, the normal derivative of the eigenfunction is obtained as Lagrange multipliers that are used to enforce Dirichlet boundary conditions in the finite element method. 


\section{CONCLUSION}

In a shape optimization problem specified by the objective function in equation (1.4), the location of the boundary $S$ is treated as an independent variation. Using the calculus of moving surfaces, we derived the first variation presented in equation (3.3), which led to the equilibrium equation (3.4). This equation is highly nonlinear as the location of the interface on which it is to be satisfied is unknown. One way to discover solutions to this equation is by numerically solving the evolution equation (8.1). This is what we did in the two-dimensional case. Alternatively, one can apply a general black box optimizer directly to the objective function (1.4).

The stability properties of equilibrium configurations with respect to smooth perturbations are revealed by the second variation of the objective function evaluated in the vicinity of the equilibrium. The equilibrium equation (3.4) is easily satisfied by spherically symmetric shapes by adjusting the radius. Radially stable equilibrium configurations may still be unstable with respect to nonradial perturbations and that is why fully morphological stability analysis is needed. We performed the stability analysis and were able to derive closed form stability criteria for all radially stable spherical configurations. The analysis yielded the surprising result that all but the lowest radially symmetric configurations are unstable with respect to the third and, in some cases, other spherical harmonics.

\section{REFERENCES}

1. J. Classen, S.-K. Su, M. Mohazzab, and H. Maris (1998). Electrons and cavitation in liquid helium. Phys. Rev. B. 57:3000-3010.

2. M. Grinfeld. (1991). Thermodynamic Methods in the Theory of Heterogeneous Systems. Longman, New York.

3. P. Grinfeld (submitted). Hamiltonian dynamic equations for fluid films. Submitted to SIAM Review.

4. P. Grinfeld and H. Kojima (2003). Instability of the $2 \mathrm{~S}$ electron bubbles. Phys. Rev. Lett. 91(10): 105301 .

5. P. Grinfeld and G. Strang (2004). Laplace eigenvalues on polygons. Computers and Mathematics with Applications 48:1121-1133.

6. P. Grinfeld and J. Wisdom (2006). A way to compute the gravitational potential for nearspherical geometries. Quart. Appl. Math. 64(2):229-252.

7. J. Hadamard (1903). Leons sur la Propagation des Ondes et les Equations de l'Hydrodynamique. Hermann, Paris.

8. J. Hadamard (1908). Memoire sur la probleme d'analyse relative a l'equilibre des plaques elastiques encastrees. Memoires presentes a l'Academie des Sciences 33:23-27.

9. J. Hadamard (1920). Comptes Rendus. Acad. Sci. 170:355-359.

10. D. Konstantinov and H.J. Maris (2003). Detection of excited-state electron bubbles in superfluid helium. Phys. Rev. Lett. 90:25302.

11. L.D. Landau and E. Lifshitz (1997). Quantum Mechanics. Butterworth-Heinemann, Stoneham, MA.

12. H.J. Maris and S. Balibar (2000). Negative pressures and cavitation in liquid helium. Phys. Today 53:29-34. 
13. H.J. Maris and W. Guo (2007). Calculation of the shape of S-state electron bubbles in liquid helium. J. Low Temp. Phys. 148:207211.

14. A.J. McConnell (1957). Applications of Tensor Analysis. Dover, New York.

15. A. Migdal (1977). Qualitative Methods in Quantum Theory. Perseus, New York.

16. T. Thomas (1957). Extended compatibility conditions for the study of surface of discontinuity in continuum mechanics. J. Math. Mech. 6:311-322.

17. T. Thomas (1961). Plastic Flow and Fracture in Solids. Academic Press, New York.

18. C. Truesdell and R. Toupin (1960). The Classical Field Theories. Springer, Berlin.

19. G.N. Watson (1995). A Treatise on the Theory of Bessel Functions. 2nd ed. Cambridge University Press, Cambridge, UK. 